\title{
Bioanalytical/Biosensors
}

\section{Biochemical Applications of Chromatography/SIMS}

\author{
K. L. Busch, M. S. Stanley, K. L. Duffin, \\ and J. C. Dunphy \\ Department of Chemistry \\ Indiana University \\ Bloomington, IN 47405
}

The balanced capabilities of a high resolution separation method coupled with a flexible detection method such as mass spectrometry results in powerful analytical methods including gas chromatography/mass spectrometry and liquid chromatography/mass spectrometry. However, separations for diverse applications such as organic synthesis, pharmacological analysis, and biochemical separations are still based on static chromatography, defined as a method in which the separation of sample components is based on a spatial separation, rather than on a difference in retention times. Although capabilities for separation of very complex mixtures for static chromatography (thin layer or paper chromatography or electrophoresis) do not rival the very high resolution achievable with the gas and liquid chromatography, the resolution is adequate for a large number of applications, and the ease and low relative cost of the methods are advantageous. General detection methods for organic compounds separated by these forms of chromatography still seem relatively crude. Color development, charring, staining, or fluorescence measurements provide the location of sample spots, but little information about sample identity. In many cases, sample identity is suggested on the basis of an $R_{\mathrm{f}}$ identical with that of the standard. Such a match is a necessary but not a sufficient means of compound identification.

Over the past 2 years, we have constructed a secondary ion mass spectrometer (SIMS) that accommodates large chromatograms within the source, and sputters organic ions from the surfaces to produce the mass spectrum of samples directly from the chromatogram. Details of instrument construction have been reported [1]. In essence, the chromatogram is moved in the $x$ and $y$ axes into and out of the point of instrument focus, defined as the locus of the primary ion beam, and the secondary ion extraction optics of a quadrupole mass spectrometer. Data in four dimensions are generated: $x, y, m / z$ ratio, and relative abundances of the ions in the mass spectrum [2-4].

Analytical advantages of the chromatography/ SIMS instrument include an independent access order to sample spots, variable integration time for spectral measurement, variable spatial resolution adjusted in real time to maximize analysis efficiency, the ability to store sample as well as data generated in its analysis, preservation of all sample applied to the chromatogram, the ability to analyze chromatograms from outside sources, and the ability to calibrate the instrument with each chromatogram [5]. We have demonstrated application of chromatography/SIMS to a variety of biochemical samples and have used the mass spectral data to identify compounds contained in the chromatogram, and to increase the resolution of the chromatographic separation. The mass spectrometer is a "biosensor" that can either be operated as a general detector, or in concert with functionalgroup-specific-derivatization reactions that have been concurrently developed.

Figure 1 illustrates part of the data array measured in an analysis of a mixture of chenodeoxy. cholic acid (A) and cholic acid (B) separated by thin layer chromatography (metal-backed silica gel plate, $20 \mu \mathrm{m}$ thickness). A dominant ion in the positive ion SIMS of chenodeoxycholic acid appears at $m / z 357$; similarly, the SIMS spectrum of cholic acid contains an abundant ion at $m / z 355$. The mass spectral data acquired over the range of $x$ and $y$ coordinates is reconstructed for ions of these masses. These data were acquired with a cesium ion gun with moderate spatial resolution. The maximum sample spot diameter is $1 \mathrm{~mm}$, and $5 \mu \mathrm{g}$ of each compound were present in the spot. Spectra could be recorded for several hours in each analysis (sample consumption rate is a few tens of picograms per second), and the same distribution is recorded if the chromatogram is removed from the mass spectrometer, stored, and reanalyzed later. 
Figure 2 represents the analysis of pyridostigmine bromide separated by thin layer chromatography. The structure of this drug is shown; the SIMS spectrum contains an abundant ion for the intact cation at $\mathrm{m} / z$ 223. Variation of this particular ion with $x$ and $y$ is shown in the abundance plot. The same data are shown plotted as isoabundance contours. Each ion in the SIMS spectrum derived from the sample exhibits similar contours, and pattern recognition programs can be used to link such ions together to form a mass spectrum of the compound free of background.

The chromatography/SIMS method has been extended to the indirect analysis of electrophoretograms. Although aqueous gels can be dried for analysis, this often degrades the spatial resolution of the separation and makes the gel difficult to handle. The high vapor pressure of water (even at low temperatures) precludes direct analysis of electrophoretic gels by SIMS. Standard sample transfer techniques such as Southern and Western blots are used to transfer samples to nitrocellulose and diazotized media, then analyzed by SIMS. Bradykinin and a series of related peptides have been so separated and analyzed; the SIMS spectra obtained are identical to those obtained from a discrete sample.

Control of the physical and chemical nature of the chromatographic surface underlies the success of chromatography/SIMS. For maximum sensitivity, the sample should be in an ionic state, and should exhibit surfactant properties in the phase transition matrix used for the analysis [4]. Figure 3 is the positive ion SIMS spectrum of a mixture of five pyrylium salts ( $10 \mu \mathrm{g}$ each) used for derivatization of primary amine groups in peptides. The recorded spectrum contains only ions from the pyrylium salt of the structure shown, which was synthesized specifically for its surface activity. No ions from the other pyrylium salts, or from the matrix, are observed. Several peptides have been derivatized with this surface-active agent. In addition to increased sensitivity, determination of the sequence of the peptide by MS/MS analysis of the parent ion of the derivative is greatly simplified.

\section{Acknowledgments}

Our research in chromatography/SIMS has been supported by the Whitaker Foundation, NIH, and NSF. The data on pyridostigmine bromide is derived from a collaborative study with Adam
Vincze of the Israel Institute for Biological Research.

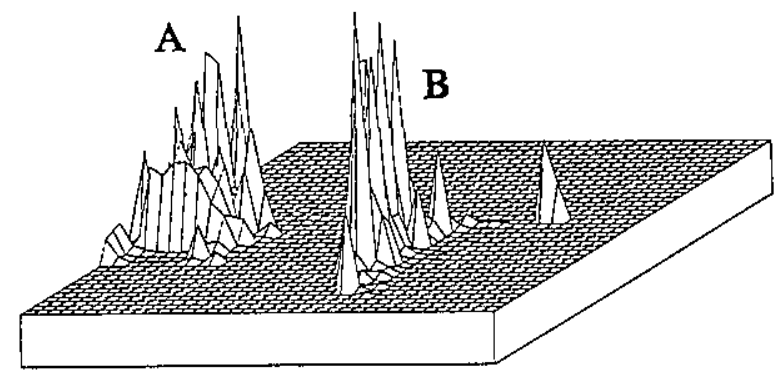

Figure 1. Reconstruction of the abundances of the ions at $\mathrm{m} / \mathrm{z}$ 357 (chenodeoxycholic acid, A) and $m / z 355$ (cholic acid, B) as a function of $x$ and $y$ coordinates of the thin layer chromatogram on which they were separated.

PYRIDOSTIGMINE BROMIDE TLC/SORBITOL
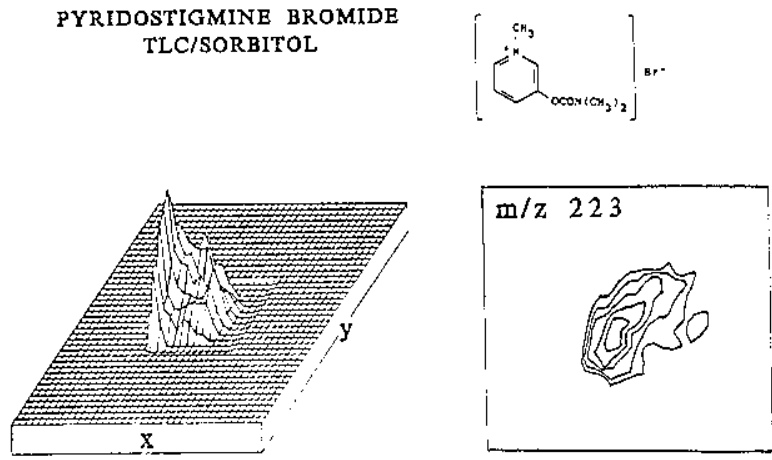

Figure 2. Abundance and isoabundance contour plots of the ion at $\mathrm{m} / \mathrm{z} 223$ corresponding to the intact cation of pyridostigmine bromide, separated from related drugs in a silica gel thin layer chromatogram.

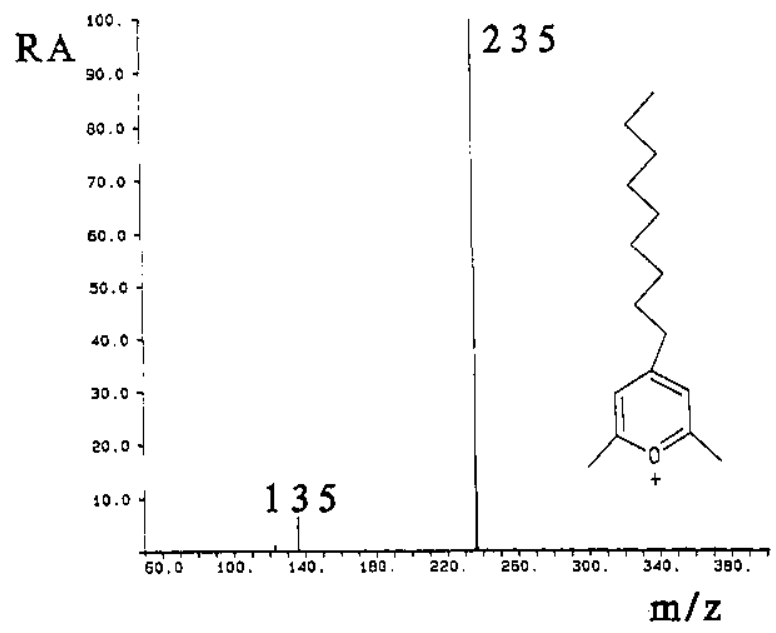

Figure 3. Positive ion secondary ion mass spectrum of a mixture of five pyrylium salts of equal concentration, showing only the ions corresponding to the pyrylium salt of the structure shown, illustrating the surfactant properties of this salt in the matrix used. 


\section{References}

[1] Fiola, J. W., DiDonato, G. C., and Busch, K. L., Rev. Sci. Instrum. 57, 2294 (1986).

[2] Busch, K. L., Fiola, J. W., DiDonato, G. C., Flurer, R. A., and Kroha, K. J., Adv. Mass Spectrom. 10, 855 (1986).

[3] DiDonato, G. C., and Busch, K. L., Anal. Chem. 58, 3231 (1986).

[4] Stanley, M. S., and Busch, K. L., Anal. Chim. Acta 194, 199 (1987).

[5] Busch, K. L., TrAC 6, 95 (1987).

[6] Stanley, M. S., Duffin, K. L., Doherty, S. J., and Busch, K. L., Anal. Chim. Acta, in press.

[7] Duffin, K. L., and Busch, K. L., manuscript in preparation.

\section{Chemiluminescence Detection in Flowing Streams-Immobilized and Solid-State Reagents}

\author{
Timothy A. Nieman \\ Department of Chemistry \\ University of Illinois \\ 1209 W. California St. \\ Urbana, IL 61801
}

Chemiluminescence (CL) reactions yield light as one of their products. Luminol (5-amino-2,3-dihydro-1,4-phthalazinedione) is one of the CL reagents most commonly used in analysis. In aqueous alkaline solution, luminol is oxidized to yield 3aminophthalate and light. Perhaps the most analytically useful oxidant is hydrogen peroxide, for which a reaction catalyst is required. Typical catalysts are peroxidase, hemin, transition metal ions, or ferricyanide. The luminol CL system most often used consists of luminol $+\mathrm{H}_{2} \mathrm{O}_{2}+$ catalyst $+\mathrm{OH}^{-}$. Measurements of $\mathrm{CL}$ intensity can be used to quantitate any of these species. Thus, luminol CL has been used to determine catalysts or species labelled with catalysts, peroxide, or species which can be converted into peroxide, and luminol, or species labelled with luminol.

For quantitative determination of any one species involved in this reaction, one needs a way to deliver the other reaction components into the reaction zone or vessel. In flowing stream situations, either flow injection analysis or liquid chromatography, one would have a stream of analyte into which were flowed streams of the other necessary reagents as shown in figure 1 . If the analyte were peroxide, then reagents 1 and 2 would be luminol and catalyst.

Such a system requires solutions of these reagents plus the necessary pumps, tubing, and mixers to deliver them and results in dilution and broadening of the sample plug. Our research effort has explored various ways to contain these reagent components in solid-state or immobilized format so that the usual reagents solutions, pumping, and tubing can be eliminated, and the reagents incorporated in-line as indicated in figure 2.

Immobilized luminol is of use for determinations of catalyst species or for determination of peroxide (and species such as glucose, cholesterol, etc., which can be enzymatically converted into an equivalent amount of peroxide). We have covalently bound luminol to silica, controlled-pore glass, and Ambersorb particles via various silane and linkage molecules. By using glutaraldehyde to bridge between the amine group on luminol and the amine group on an aminoalkylsilane, loadings of $15 \mathrm{mg}$ luminol/g support are achieved for supports with about 400 square meters surface area/ gram. This material is packed into a column placed into the flow stream. The immobilized luminol is stable in neutral solution, but injection of a portion of an alkaline solution will release a controlled amount of luminol (via hydrolysis of the bond between luminol and glutaraldehyde); the amount released is dictated by the $\mathrm{pH}$ and volume of the solution injected. We have also contained luminol simply by adsorption onto Ambersorb and released it in the same manner. Advantages to release of luminol prior to the CL reaction include improvement in the CL quantum efficiency and increased flexibility in engineering the contact with the other reagents. By use of this material in flow injection, a detection limit of $0.1 \mu \mathrm{M}$ for peroxide is obtained.

An immobilized catalyst is of use for determination of peroxide (and analytes enzymatically converted to peroxide) or analytes that have been labeled with luminol or related compounds. An approach that we have investigated involves use of electrogeneration of luminol CL. Luminol can be oxidized at a positively-biased electrode, and CL results if oxygen or hydrogen peroxide are present. Electrogeneration of luminol $\mathrm{CL}$ offers the unique characteristics that the CL emission is confined to near the electrode surface and that the reaction can be turned "on" and "off" via control of electrode potential. The net electrogenerated CL (ECL) intensity due to peroxide injections appears as peaks on top of an appreciable and inescapable back- 